\title{
INFLUENCE OF STRESS CONDITION ON ADIABATIC SHEAR LOCALIZATION OF TUNGSTEN HEAVY ALLOYS
}

\section{ZHIGANG WEI, JILIN YU, JIANRONG LI, YONGCHI LI and SHISHENG HU}

\author{
Department of Modern Mechanics, University of Science and Technology of China \\ Hefei, Anhui 230027, P. R. China
}

\begin{abstract}
Dynamic deformation and failure behavior of a tungsten heavy alloy (93W) under complex stress condition are studied using a split Hopkinson pressure bar (SHPB) apparatus. Cylindrical, step-cylindrical and truncated-conic specimens are used to generate different stress condition in an attempt to induce strain localization in the alloy. The microstructure of the specimens after tests is examined by optical microscopy and scanning electronic microscopy (SEM). It is found that in all the specimens, except the cylindrical ones, intense strain localization in the form of shear bands is initiated at stress concentration sites. In order to analyze the stress condition of different specimen geometry, finite element simulations are also presented. The Johnson-Cook model is employed to simulate the thermo-viscoplastic response of the material. It is found that dynamic deformation and failure modes are strongly dependent on the geometry of the specimens. The stress condition controlled by specimen geometry has significant influence on the tendency for shear band formation. The adiabatic shear band has general trends to initiate and propagate along the direction of maximum shear stress. It is suggested that further studies on the control of the stress condition to promote shear band formation be conducted in order to improve the penetration performance of the tungsten heavy alloy. 2001 Elsevier Science Ltd. All rights reserved.
\end{abstract}

Keywords: adiabatic shear localization, tungsten heavy alloys, dynamic behavior

\section{INTRODUCTION}

Tungsten heavy alloys (WHA) such as $93 \mathrm{~W}-\mathrm{Ni}-\mathrm{Fe}$ are widely used in penetrators due to their high density and strength as well as good ductility. Adiabatic shear localization plays a key role in penetration performance at conventional ordnance velocities. It is well known that the "mushrooming" effect deteriorates the penetration performance, while the "self-sharpening" effect improves the performance. Magness and Farrand ${ }^{[1]}$ explained this self-sharpening effect in terms of adiabatic shear bands. The localization of plastic flow in shear bands, which frequently leads to fracture of material, is usually attributed to the instability resulting from thermal softening which overcomes the effect of strain hardening in a heavy deformed region ${ }^{[2]}$.

It is believed that increasing launch velocity $(>2000 \mathrm{~m} / \mathrm{s})$ is one of the most important approaches to improve the penetration depth in the future. Thus, the investigation of deformation, failure mechanisms and penetration performance of tungsten heavy alloys at high velocities is critically needed ${ }^{[3]}$. At a velocity substantially above conventional ordnance ones, disintegration behavior is considerably more complex. Tensile fracture and shear localization may be accompanied or even supplanted by two additional disintegration mechanism, namely melting 
and vaporization ${ }^{[4,5]}$. However, experiments have shown that adiabatic shear bands also play an important role in the disintegration at hypervelocity impact. As shown by Shockey et al. ${ }^{[S]}$ in their rod-plate impact experiments, the mushroom shape of the impacted end of a 4340 steel rod is caused by shear bands, as well as homogeneous plastic flow and tensile fracture. Debris material adjacent to the interface between the rod and the plate was in a form of solid fragments due to intersecting shear bands.

The penetration performance of DU alloy is superior to that of a tungsten alloy under the same conditions due to the "self-sharpening" effect. However, the use of DU alloy poses serious problems regarding environmental pollution and health care. Therefore, many studies have recently been done on ways to improve the penetration performance of tungsten heavy alloy penetrators. Unfortunately, tungsten heavy alloys are found to be insensitive to adiabatic shear failure when subjected to high strain rate compressive deformation under uniaxial conditions and exhibit less "self-sharpening" effect in penetration ${ }^{(1,6]}$. Hence, most current studies have been focused on developing new types of WHA that are more effective on the battlefield through controlling its microstructure to promote shear band formation. Two basic methods are pursued ${ }^{[7]}$. One method is a flow-softening approach, such as modifications or replacement of the nickelbase matrix in conventional WHA ${ }^{[8]}$. The other method is a flow-anisotropy approach, attempts to develop a directional flow-softening behavior observed for oriented single-crystal ${ }^{[9]}$ and oriented columnar-grained ${ }^{[10]}$ tungsten penetrators via crystallo-graphic orientation of the tungsten phase in the composites. Recently, it was found that pre-twisted WHA penetrators show better ballistic performance than that of un-twisted ones. The reason can also be attributed to the "self-sharpening" effect ${ }^{[6]}$.

A lot of mechanical behavior data are available for WHA under dynamic loading. Most of these data were obtained under uniaxial compressive or tensile conditions or under simple shear conditions. A few mechanical and failure behavior data are available for these alloys under combined compression/shear loading conditions ${ }^{[1-13]}$. However, in a high velocity penetration process, the stress condition close to the surface of the penetrator nose is more complicated. Accordingly, in order to understand the mechanism of self-sharpening effect and improve the ballistic performance of tungsten heavy alloy penetrators, the dependence of adiabatic shear localization on stress conditions at high strain rates is of fundamental importance.

Dick et al. ${ }^{[4]}$ conducted reverse ballistic impact tests on tungsten heavy alloy rods and examined the deformation and failure mechanisms of the rods. A numerical simulation of their tests was conducted by Stevens and Batra ${ }^{[15]}$ recently. Meyer et al. ${ }^{[16]}$, Wei et al. ${ }^{[1]]}$ studied adiabatic shear banding in inclined cylindrical WHA specimens at strain rates of $10^{2} \mathrm{~s}^{-1}$ and $10^{3} \mathrm{~s}$ ', respectively. It should be mentioned that the shear bands, in general, occurred along a diagonal line of the cross-section in uniaxial compression tests ${ }^{[17]}$, or along the minor diagonal in compression/shear impact (inclined specimen) ${ }^{[1,16]}$. Only two halves separated by a planar shear band may be formed in the failure of the specimen in these experiments.

It is the intent of this paper to provide a description toward a coherent understanding of the sensitivity of the deformation and failure of WHA to the stress conditions of specimens. This study demonstrates the microscopic mechanism of deformation and failure in tungsten heavy alloys under complex stress conditions, and it is hoped that such investigation will provide an insight into the way to improve the ballistic performance of the WHA penetrators. 


\section{EXPERIMENTS}

\section{Materials and Experimental Setup}

The W-Ni-Fe heavy alloy used in this work contains $93 \%$ of tungsten by weight with a nickel to iron ratio of $7: 3$. The microstructure of the original tungsten alloy consists of nearly spherical tungsten grains (typically $30 \sim 60 \mu \mathrm{m}$ in diameter) surrounded by Ni-Fe matrix.

In order to investigate the influence of the stress condition, cylindrical, step-cylindrical and truncated-conic specimens are used for comparison. A Split Hopkinson pressure bar (SHPB) is used to load samples at strain rates of about $10^{3} \mathrm{~s}^{-1}$. A schematic drawing of the SHPB apparatus and the geometry of the specimens are shown in Fig.1. After SHPB tests, some intact specimens are precisely cut along the axial direction. These surfaces are then polished, etched, and then characterized using the optical microscopy to examine the deformation and fracture behavior of the material. Scanning electron microscope studies are performed on the fractured surface of the specimens after impact tests in order to identify the characteristics of fracture and its mechanism.

\section{Uniaxial Dynamic Compression of Cylindrical Specimens}

Experiments show that adiabatic shear banding is very hard to find in uniaxial compression with cylindrical specimens. The optical micrograph of the deformed specimen shows that originally spherical W-grains have been deformed to an ellipsoidal shape due to compression. No shear bands and microcracks were observed even at high strain rates (as high as $5000 \mathrm{~s}^{-1}$ ) and axial engineering strains (as high as $60 \%$ ).

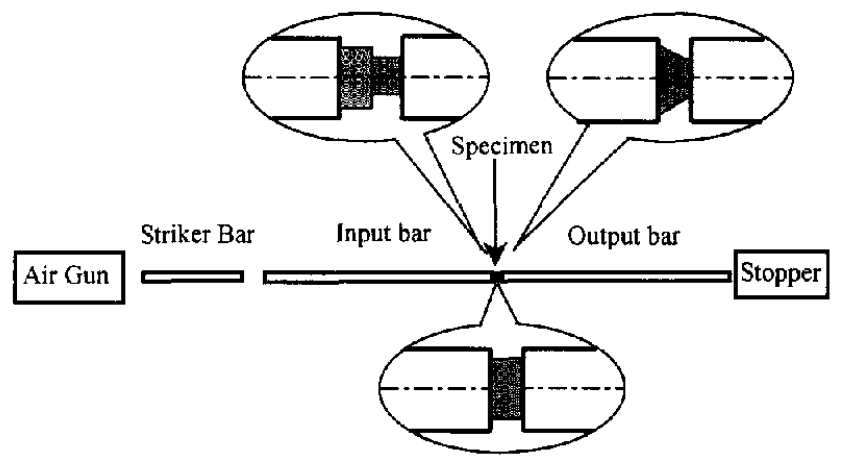

Fig.1. Sketch of the split Hopkinson pressure bar apparatus and specimen geometry.

\section{Dynamic Compression of Step-Cylindrical Specimens}

It is found that step-cylindrical specimens were very sensitive to shear banding. A pair of heavily localized deformation bands, situated symmetrically with respect to the axes, is formed after an impact with very low kinetic energy, when a specimen undergoes only small plastic deformation that the axial length decreases from $4.5 \mathrm{~mm}$ to $4.3 \mathrm{~mm}$, as shown in Fig.2.

Under higher impact energy with larger plastic deformation, a narrow adiabatic shear band within specimens is observed, as shown in Fig.3. Near the shear band, grains were highly distorted and elongated. It should be noted that in the specimen only one annular shear band is formed. Within the band the tungsten grains are heavily deformed, and pulled into long stringers along the shear direction. Presumably the adiabatic heating there is sufficient to soften the 
tungsten grains and develop large plastic deformation. Away from the shear band the tungsten grains rotate themselves sufficiently to accommodate the shear deformation. No tensile microcrack accompanying the shear deformation is observed away from the band at extremely large shear strains. Fig. 3 also shows a narrow shear crack indicating that the adiabatic shear band is the precursor of macro-break of the specimen.

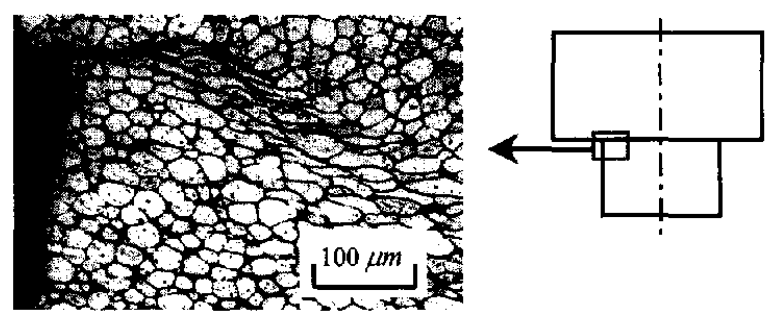

Fig.2. Adiabatic shear band at small deformation in a step cylindrical specimen.

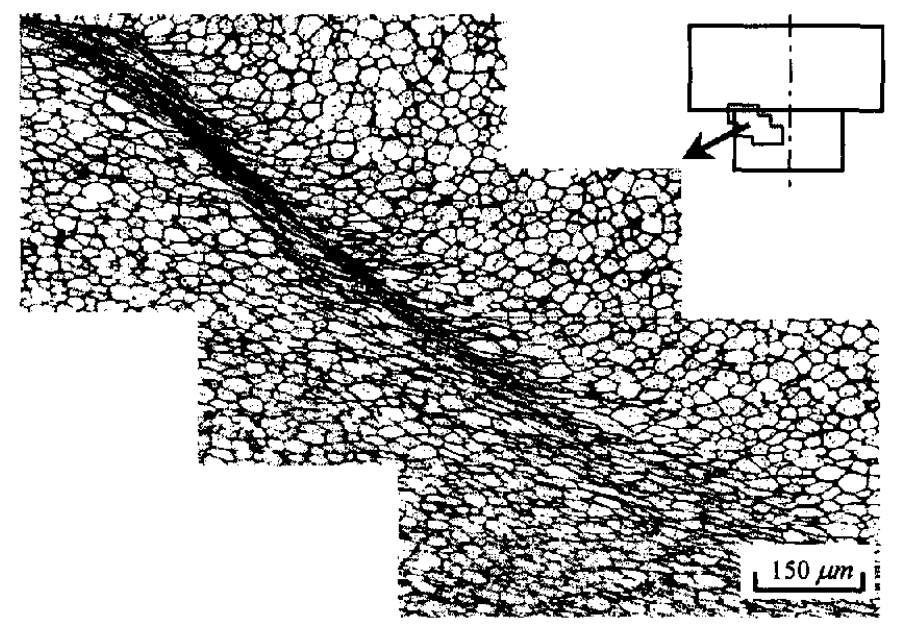

Fig.3. Adiabatic shear band at large deformation in a step cylindrical specimen.

\section{Dynamic Compression of Truncated-Conic Specimens}

Intact Specimens. Some impact tests with lower kinetic energy were conducted on truncated-conic specimens. The longitudinal deformation is small, from originally $5 \mathrm{~mm}$ to 4.5 $\mathrm{mm}$. A severe localized deformation band where tungsten grains are significantly elongated can be found by optical microscope, indicating a developing adiabatic shear band, as shown in Fig.4. The shear band is situated symmetrically with respect to the axes, initiated from the blunt comer of the truncated specimen and propagated into the bulk downward to the bottom edge. Within the shear band no microcracking is observed in the tungsten grains. Only one shear band is formed. About $50 \mu \mathrm{m}$ away from the center of the shear band, tungsten grains are rarely deformed. Fig. 5 is a photograph of an intact specimen with only two cracks along the radial direction due to hoop deformation. However, Inhomogeneous shear deformation was not observed by visual 
inspection.

Break-up Specimens. To further investigate the adiabatic shear localization and failure mechanism, some impact tests with higher kinetic energy were conducted. The material failed at about $20 \%$ compressive deformation. Fig. 6 is a photograph of the fully fractured specimen. The specimen has broken into a few fragments. One is in the shape of a reversed truncated cone formed through shear fracture. Surrounding it, the outside ring is broken into several pieces due to radial cracking.

Fig.7(a) and Fig.7(b) show fractographs of the tensile fracture and shear fracture surfaces, respectively. The tensile fracture surface shown in Fig.7(a) is mostly intergranular. Tungsten grains there are much less deformed than those near the shear fracture surface, indicating the brittle nature of the material under tensile loading. By contrast, the main feature of the shear fracture surface, Fig.7(b), is the rupture of highly deformed W-grains. This is in accord with the optical observation shown in Fig.4. Fig.7(c) shows a fractograph of the transition zone between the tensile ruptured surface and the shear ruptured surface. It is observed that the shear-fractured surface is much smoother than the tensile fracture surface.

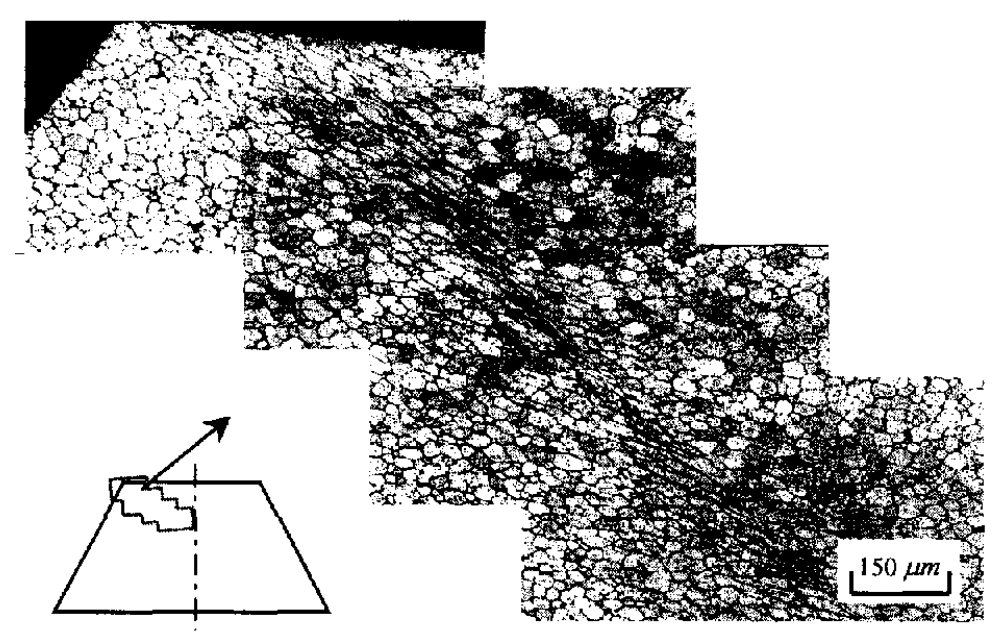

Fig.4. Adiabatic shear band in a truncated-conic specimen with small deformation.

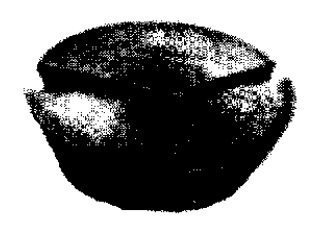

Fig.5. Tensile fracture pattern of a specimen after low energy impact.

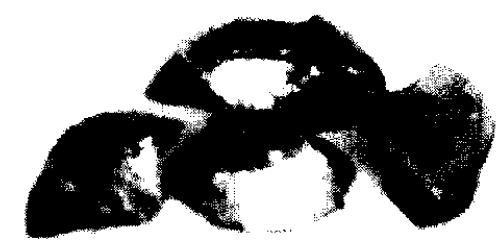

Fig.6. Shear and tensile fractured specimen after high energy impact. 


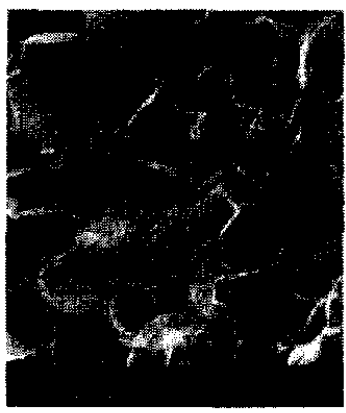

(a)

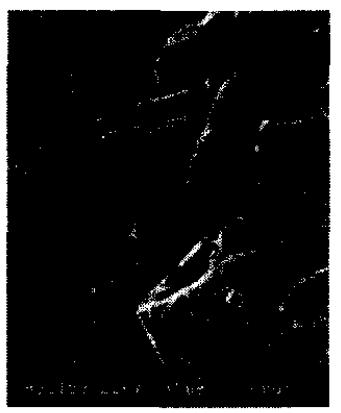

(b)

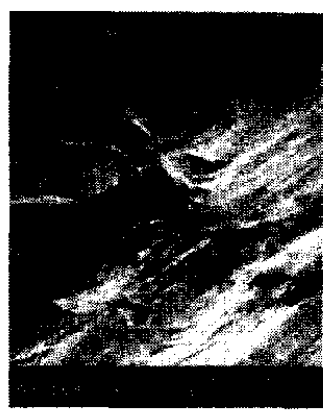

(c)

Fig.7. Fractographs of the (a) tensile fracture surface, (b) shear fracture surface and (c) transition zone in Fig.6.

\section{NUMERICAL ANALYSIS}

\section{Formulation of the Problem}

The finite element method (as implemented within ABAQUS) is used to calculate the overall response of the specimens under dynamic compression. Only cylindrical and truncated-conic specimens are considered here for comparison. Axisymmetric elements are used. The initial mesh for the cylinder specimen consists of 50 and 40 quadrilateral elements in radial direction and axial direction, respectively. However, the truncated-conic specimen is expected to deform more severely. Hence triangular elements, which are suitable in cases of severe distortions, are used. The initial mesh for the truncated-conic specimen consists of 100 and 40 triangle elements in radial direction and axial direction, respectively. No failure or fracture criterion is considered, thus each constituent can undergo unlimited amount of plastic deformation.

The height and diameter of the cylindrical specimen are $4 \mathrm{~mm}$ and $7 \mathrm{~mm}$, respectively. The truncated-conic specimen has the same height. The diameters of its two surfaces are $6 \mathrm{~mm}$ and 9 $\mathrm{mm}$. The specimen is initially at rest, stress free and at a uniform temperature. The top and bottom surfaces are assumed friction free with velocity control. The magnitude of the predescribed velocity increases from zero to $8 \mathrm{~m} / \mathrm{s}$ in $5 \mu$ s and then remains fixed at $8 \mathrm{~m} / \mathrm{s}$.

The rate-dependent thermo-viscoplastic model of Johnson-Cook ${ }^{[18]}$ is used for numerical simulation.

$$
\sigma=\left(A+B \varepsilon^{n}\right)\left(1+C \ln \frac{\dot{\varepsilon}}{\dot{\varepsilon}_{0}}\right)\left[1-\left(T^{*}\right)^{m}\right]
$$

where

$$
T^{*}=\frac{T-T_{r}}{T_{m}-T_{r}}
$$

and $\dot{\varepsilon}_{0}=10^{-4} \mathrm{~s}^{-1}$ is the reference strain rate, $T_{m}$ the melting temperature of the material, and $A, B$,

$n, C$ and $m$ are material constants.

It is assumed that the effect of heat conduction is neglectable so the response is adiabatic. 
Thus the local temperature rise depends on the work of deformation by

$$
d T=\frac{0.9}{\rho c_{p}} \sigma d \varepsilon
$$

The material constants used in this simulation can be found in the Table 1 .

Table 1. Material parameters for WHA. ${ }^{[2]}$

\begin{tabular}{cr}
\hline Parameter & Value \\
\hline $\mathrm{A}$ & $1093 \mathrm{MPa}$ \\
$\mathrm{B}$ & $1270 \mathrm{MPa}$ \\
$\mathrm{C}$ & $18.8 \times 10^{-3}$ \\
$\mathrm{n}$ & 0.42 \\
$\mathrm{~m}$ & 0.78 \\
$\mathrm{~T}_{\mathrm{r}}$ & $293 \mathrm{~K}$ \\
$\mathrm{~T}_{\mathrm{m}}$ & $1850 \mathrm{~K}$ \\
$c_{p}$ & $134 \mathrm{~J} / \mathrm{kg} \cdot \mathrm{K}$ \\
$\rho$ & $18600 \mathrm{~kg} / \mathrm{m}^{3}$ \\
\hline
\end{tabular}

\section{Results and Discussion}

It transpires from the numerical results that, even at very large compressive deformation (about $50 \%$ ), the deformation of the cylindrical specimen is still uniform. No shear localization appears.

Fig. 8 presents the distribution of Tresca stress in the truncated-conic specimen after a height reduction of $1 \%$. It is clear that the stress is inhomogeneous. Fig. 9 and Fig. 10 show contours of the equivalent plastic strain $\varepsilon_{e q}^{p}$ and shear strain $\varepsilon_{r z}$ in the truncated-conic specimen at $10 \%$ height reduction. A dominant narrow region of intense plastic shear deformation stands out clearly in these figures. These results show that shear deformation concentration occurs even at small deformation.

In order to delineate the variation of the deformation process, we have plotted in Fig.11 the time history of $\varepsilon_{e q}^{p}$ at five different positions, i.e., B, C, D near the shear localization region and A, E far away from the region shown in Fig. 8. It is clear that $\varepsilon_{e q}^{p}$ within the band is much higher as compared with that at the neighboring points. The deformation increases rapidly near the shear band but rather gradually in neighboring points.

In conclusion, the numerical results have indicated that different configurations give quite different deformation patterns. For cylindrical specimens no shear bands are observed even at a height reduction of $50 \%$. However, for the truncated conic specimens, shear localization initiates easily from the stress concentration sites and a mature shear band is formed even at small height reduction. These results are in agreement with our experiments. This signifies that the local stress condition plays a critical role in the shear localization.

It should be mentioned that a fully developed adiabatic shear band can not be simulated in our calculation due to the coarse mesh. 


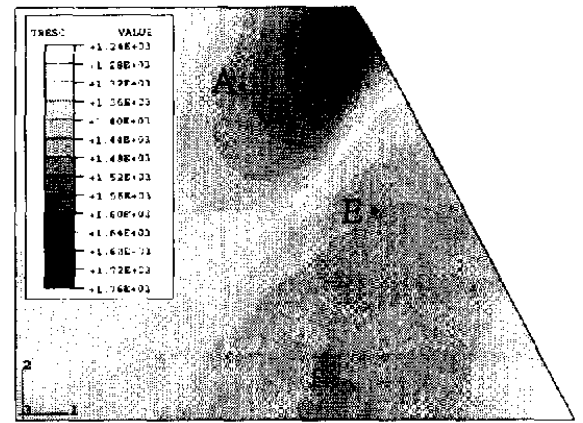

Fig.8. Distribution of the Tresca stress in the truncated-conic cylinder

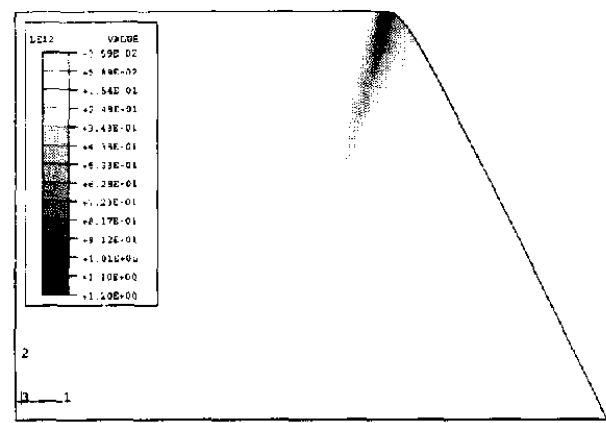

Fig.10. Contours of the $r z$-component of the strain at $10 \%$ height reduction.

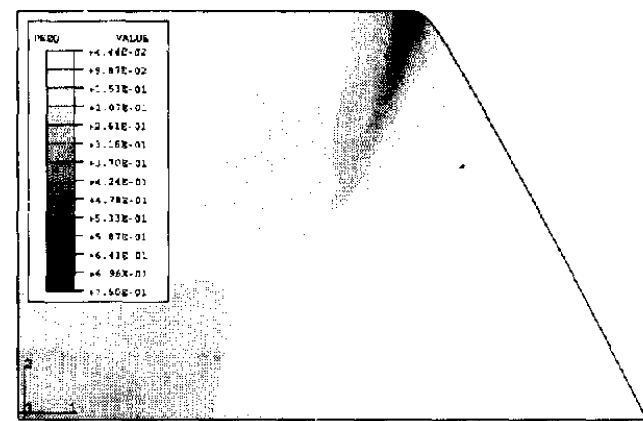

Fig.9. Contours of the equivalent plastic strain in the truncated-conic cylinder

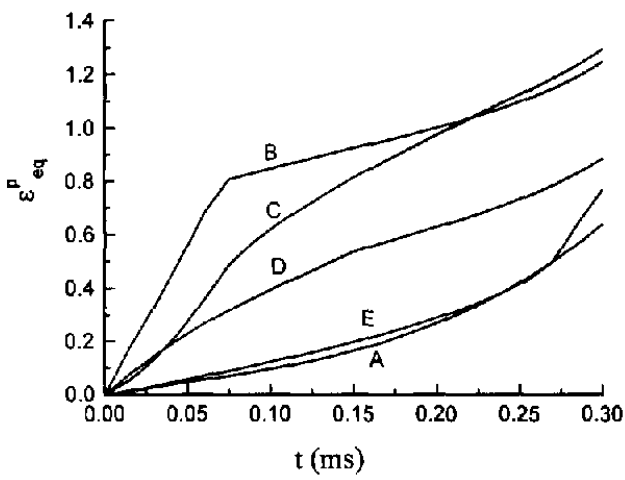

Fig.11. The time history of the equivalent strain at various points

\section{DISCUSSION}

Tungsten heavy alloy, in nature, has low sensitivity to adiabatic shear localization. However, it is worth to pointing out that adiabatic shear localization is sensitive to stress condition. Comparing the deformation and failure behavior of cylindrical specimens with the stepcylindrical and truncated-conic specimens, the difference is evident. The above results indicate that the deformation and failure modes are significantly dependent on the stress condition, which is controlled by geometry of specimens.

When a cylindrical specimen is under dynamic uniaxial compression, there is no favorite direction of shear deformation and shear localization will hardly take place. Microstructures, such as hard tungsten grains and softer matrix, have little effect on the nucleation of adiabatic shear band and their effects must be secondary to that of stress condition.

The influence of stress condition on shear localization in tungsten heavy alloys has also been confirmed by other investigations where shear stress concentration were introduced by complex loading conditions ${ }^{[1,16]}$. 
Ramesh $^{[19]}$ examined the failure of a W composite during dynamic shear loading with a torsional SHPB bar, and noted that adiabatic shear bands were formed relatively easier than under dynamic compressive condition, and the localized shearing failures were accompanied by considerable microcracks on either side of the shear bands. Zhou et al. ${ }^{[13]}$ conducted a compression/shear plate impact experiment on $\mathrm{W}$ composite, they observed adiabatic shear bands only at extremely large shear strains (larger than unity) but no microcrack at all. Yadav and Ramesh hypothesized that the adiabatic shear localization can be triggered by damage and superimposition of pressure would suppress the tensile damage mechanism, as observed in the compression/shear case ${ }^{[12]}$.

Based on the experimental results of this study, adiabatic shear banding occurs easily under complex stress conditions, such as in step-cylindrical and truncated-conic specimens. However, no microcrack was observed in tungsten grains before the shear band forms, while the deformation process seems to be dominated mainly by tungsten grains. The influence of microcracking on the generation and development of all adiabatic shear bands seems minor in the case studied.

Furthermore, there is no evidence that the compressive load would suppress the adiabatic shear instability, when one compares the response of the cylindrical and step-cylindrical specimens. Compressive load occurs in both cases but adiabatic shear band takes place only in the step-cylindrical specimens. It seems that compressive load would only influence the formation of microcracks.

For real WHA penetrators, adiabatic shear bands appear only after the development of a large mushroomed head. At the early stage of deformation during penetration, axial pressure is dominant at the head of the penetrator. This pressure effectively suppresses adiabatic shear band formation in the tungsten heavy alloy penetrator at its head until the head deforms into a mushroom shape and the influence of geometry dominates the response. This is in agreement with our experimental results.

Considering the positive effect of the adiabatic shear band formation in improving the penetration performance of the WHA penetrator, it can be expected that penetration performance can be enhanced by controlling the stress condition in the penetrator at or near the interface between the penetrator and the target.

\section{CONCLUSION}

Dynamic compression tests were conducted on cylindrical, step-cylindrical and truncatedconic specimens using SHPB apparatus to study the deformation and failure behavior of tungsten heavy alloy at high strain rates. Numerical calculations are conducted to simulate the experiments. The following conclusions can be drawn from the results.

1. It is found that the adiabatic shear banding in step-cylindrical and truncated-conic specimens is formed easier than that in the case of uniaxial compression. No shear band is observed in cylindrical specimens even at very large deformation. The reason can be attributed to the complex stress conditions which triggers the initiation and development of the adiabatic shear localization.

2. Two rupture mode were formed for the truncated-conic tungsten heavy alloy cylinder subjected to dynamic compressive loading, one is tensile crack along radial direction, the other form is the adiabatic shear localization along maximum shear stress direction. 
Acknowledgement-This work is supported by the Chinese Academy of Sciences (project No. KJ0952- $\mathrm{J}_{1}-253$ ) and the National Defense Key Laboratory.

\section{REFERENCES}

[1] Magness Jr L S, Farrand T G. Deformation Behavior and its Relationship to the Penetration Performance of High-Density KE Penetrator Materials. in Proc. 1990 Army Science Conference, Durham, NC, 1990: p. 149164.

[2] Bai Y, Dodd B. Adiabatic shear Localization, Pergamon. 1992.

[3] Crowson A. Materials issues in impact engineering. In Shim V P W, Tanimura S, Lim C T, editors. Impact Response of Moterials and Structures, Oxford, Oxford University Press. 1999. p. 15-25.

[4] Herrmann W. Wilbeck J S, Review of hypervelocity penetration theories, Int. J. Impact. Engng., 1987; 5:307322.

[5] Shockey D A, Curran D R, Osher J E, Chau H H. Disintergration behavior of metal rods subject to hypervelocity impact. Int. J. Impact. Engng., 1987; 5:585-593.

[6] Wei Z G, Hu S S, Li Y C, Fan C S. Dynamic properties and ballistic performance of pre-torqued tungsten heavy alloys. In Niekerk C V, editor. Proceeding of the 17th International Symposium on Ballistics, Vol.3, South Africa, The South Africa Ballistics Organization, 1998: p. 391-398.

[7] Magness Jr L S, Kapoor D. Flow-softening tungsten composites for kinetic energy penetrator applications, In Bose A, Dowding R, editors. Proceedings of the 2nd international conference on Tungsten and Refractory Metals, Mclean, VA, 1994: p. 11-20.

[8] Dunn P S, Baker B D. Target penetrator interaction: 70 volume percent tungsten-30 volume percent uranium penetrator material. In Bose A, Dowding R, editors. Proceedings of the first International Conference on Tungsten and Tungsten Alloys, Arlington VA, Nov. 15-18, Metal Powder Industries Federation, 1992: p. 487496.

[9] Bruchey W, Horwarth E, Kingman P. Orientation dependence of deformation and penetration behavior of tungsten single crystal rods, in Crowson A, Chen E S, editors. Tungsten and Tungsten Alloys-Recent Advances, 1991: p.121.

[10] Leonard W, Magness Jr L S, Chung M, Kapoor D. The performance of oriented columnar-grained polycrystalline tungsten penetrators, in Proc. 16th International Symposium on Ballistics, San Francisco CA, 1996: p. 617-626.

[11] Wei Z G Yu J L, Hu S S, Li Y C. Influence of microstructure on adiabatic shear localization of pre-twisted tungsten heavy alloys. Int. J. Impact. Engng.,2000;24:747-758.

[12] Yadav S, Ramesh K T. The mechanical properties of tungsten-based composites at very high strain rates. Material Science and Engineering 1995; A203:140-153.

[13] Zhou M, Clifton R J. Dynamic constitutive and failure behavior of a two-phase tungsten composite. J. Appl. Mech., 1997; 64:487-494.

[14] Dick R D, Ramachandran V, Williams J D, Armstrong R W, Holt W H, Mock W Jr. Dynamic deformation of W-Ni-Fe alloy via reverse-ballistic impact. In Crowson A, Chen E S, editors. Tungsten and Tungsten Alloys Recent Advances. The Minerals, Metals and Materials Society, 1991: p. 269-276.

[15] Stevens J B, Batra R C. Adiabatic shear bands in the Taylor impact test for a WHA rod. International Journal of Plasticity 1998; 14:841-854.

[16] Meyer L W, Staskewitsch E, Burblies A. Adiabatic shear failure under biaxial dynamic compression/shear loading. Mechanics of Materials 1994; 17:203-214.

[17] Belk J A, Watson C H, Bently A R, Hogwood M C. Dynamic deformation characteristics of tungsten alloys. In Bose A, Dowding R, editors. Proceedings of the 2nd international conference on Tungstem and Refractory Metals. Mclean, VA, 1994: p. 389-399.

[18] Johnson G R, Cook W H. A constitutive model and data for metals subjected to large strain rates and high temperatures. In Proceedings of the Seventh International Symposium on Ballistics, The Hague, The Netherlands, 1983: p.541-548.

[19] Ramesh K T. On the Localization of shearing deformations in tungsten heavy alloys. Mechanics of Materials 1994; 17:165-173. 